\title{
Palliative pelvic exenteration - literature review
}

\author{
N. Bacalbașa ${ }^{1}$, A. Traistaru², Irina Bãlescu ${ }^{3}$, V. Brașoveanu ${ }^{4}$
}

Corresponding author:

Nicolae Bacalbasa

Dimitrie Racovita street, no. 2

Bucharest, Romania

E-mail: nicolae_bacalbasa@yahoo.ro

\author{
I"Carol Davila" University of Medicine and Pharmacy, Bucharest, Romania \\ 2Elias Universitar Hospital Bucharest, Romania \\ 3"Ponderas" Hospital, Bucharest, Romania \\ "Department of General Surgery and Liver Transplantation "Dan Setlacec" \\ Fundeni Clinical Institute, Bucharest, Romania
}

\begin{abstract}
Although it is one of the most aggressive surgical procedures, pelvic exenteration represents the only option for patients presenting advanced pelvic tumors. Due to the improvements of surgical technique and association of neo-adjuvant therapies, pelvic exenteration is performed nowadays rather with curative intent. However there are still patients in whom a radical procedure is no longer feasible but who present invalidating symptoms. In these cases a palliative exenteration can be performed. We present a literature review of the most important studies regarding indications and outcomes of palliative exenteration
\end{abstract}

Key words: local invasive pelvic tumors, distant metastases, palliative exenteration

Nowadays pelvic exenteration is rather a radical surgical procedure for cases presenting locally invasive pelvic tumors or pelvic recurrences after preirradiated and surgically treated cervical cancers which consists in en bloc resection of the genital organs, inferior urinary tract and rectosigmoidian resection. Other pathologies which can be successfully treated by total pelvic exenteration are locally invasive uterine cancer, vaginal or vulvar cancer. (1)

The first pelvic exenteration was performed in 1948 by Alexander Brunschwig, an American surgeon in a patient with locally invasive pelvic recurrent cervical cancer with palliative intent. After this some other patients were submitted to the same type of surgery but unfortunately, at that moment the postoperative mortality reached almost $23 \%$. However the benefits provided by this surgery even when it was done with palliative intent was expressed best by Brunschwig himself when saying: "Because of the advanced stage of their disease, it is not to be anticipated that many, if any, of these patients will survive for very prolonged periods..... On the other hand, of those surviving at this writing, not one has expressed the feeling that they would have preferred to have remained as they were and not to have had the operation". (2)

Nowadays due to the evolution of the preoperative imaging studies, due to the improvement of operative techniques and of the postoperative management and intensive care, the postoperative mortality decreased up to 3-5\% and the 5year overall survival reached up to $50 \% .(3,4)$ 
In cases presenting pelvic recurrences after pre-irradiated and surgically treated cervical tumors the only therapeutic options are palliative chemo-irradiation (associated with 1 year overall survival lesser than 15\%) or palliative pelvic exenteration. Due to the fact that usually these recurrences are limited to the pelvic space, without distant metastases, a new surgical procedure is usually recommended. (3)

\section{Indications}

Palliative pelvic exenteration is usually recommended for cases diagnosed with locally invasive recurrent cervical cancer. The palliative role is best revealed in those patients presenting locally invasion of the pelvic bones, of the pelvic vessels (common iliac vessels, external iliac vessels), disseminiated lymph node metastases or distant visceral metastases but in whom the local symptoms (urinary or digestive fistulas or obstructions, bleeding, pelvic pain) cannot be controlled throughout other therapeutic approaches. In a study conducted by Hockel on 36 patients with recurrent gynecologic malignancies ( 29 cases) and primary pelvic tumors ( 7 patients) between 1996 and 2002 the 5 year overall survival was $49 \%$ for the whole group and $46 \%$ for those in whom a palliative procedure was performed. In 34 cases a good local control of the tumor was achieved, with negative margins of resection. (5)

Other indications of palliative pelvic exenteration are locally invasive vulvar neoplasms, advanced ovarian or endometrial cancer associated with invalidating symptoms (pain, bleeding, obstruction) in which the probability of distant micrometastases is very high. The 5 year overall survival in cases with locally advanced endometrial or ovarian cancer after palliative exentration reaches up to $20-40 \%$. $(6,7)$

\section{Contraindications}

The palliative role of pelvic exenteration gave birth to a lot of controversies due to the high risk of early postoperative morbidity associated with an increased hospitalization stay and increased mortality. Absolute contraindications for performing an exenteration with radical intent (peritoneal metastases, visceral or lymph node metastases) are no longer valuables for cases presenting invalidating symptoms and who could benefit from a palliative procedure. Nowadays the main contraindication for performing a palliative procedure is related to the poor general status of the patient. The classic association between inferior member oedema and pain is pathognomonic for local invasion of the lateral pelvic wall and might constitute a formal contraindication for surgery. However in cases with lateral extension the most important factor is the location of the extent reported to the obturatory nerve. In cases in which the extent is located above the obturatory nerve resection is no longer feasible. In all the other cases a laterally extended endopelvic resection (LEER) can be performed. This surgical procedure is associated with parietal pelvic resections and muscular ersection and might have a palliative intent. (5)

\section{Preoperative evaluation of the patients}

The general biological status of the patient should be an appropriate one for supporting a long surgical procedure (between 4 and 8 hours). Sometimes preoperative preparation with parenteral nutrition and blood transfusions might be needed. Age over 65 years and the presence of associated co-morbidities increases the rate of peri-operative complications and needs a more specific preoperative evaluation and preparation.

A proper preoperative clinical exam might reveal the presence of distant metastases (supra-clavicular or inguinal lymph node metastases, cutaneous distant metastases). In these cases the surgical procedure will have a palliative intent. In other cases the local extent of the neoplastic disease might be hard to be established preoperatively due to the important structural modifications related to the pre-operative pelvic irradiation or due to the association of neoplastic cellulitis.

The idea of palliative surgery can be established preoperatively when association between local unresectable invasion or distant metastases and debilitating pelvic symptoms is found or it can be discovered intraoperatively. The latter situation can be encountered when preoperative imaging exams fail to recognize local bony invasion or distant micrometastases and one of these features are found only after a complete tumoral dissection is provided.

Preoperative imaging studies can also be associated with false results due to the high grade of local inflammation. In these cases the real limit between the tumor and the pelvic wall is very hard to be established and differentiation between tumoral invasion and local inflammation is hard to be done. The most appropriate imaging evaluation is done by MRI and has been widely studied in clinical trials.

In a retrospective study conducted by Popovich involving 23 patients with locally advanced pelvic malignancies, MRI had a negative predictive value of $100 \%$ in establishing the involvement of the pelvic wall and 95\% in revealing the presence of disseminated lymph node metastases. The lateral pelvic extent was poor estimated in 4 cases, 3 of these patients presenting un-differentiable lesions due to the pre-operative 
irradiation. The visceral invasion was correctly shown in $81 \%$ of cases for the urinary bladder invasion and in $85 \%$ of cases for the rectal invasion.(8)

Donati et al realized a study on 50 patients with persistent or recurrent pelvic tumors. The preoperative MRI had a $87 \%$ sensibility in detecting the urinary bladder invasion, $81,3 \%$ in establishing the urinary bladder involvement and $87,5 \%$ in detection of the pelvic wall extension. (9)

In order to establish the presence of distant metastases, studies have shown that the most appropriate method is PET-CT with a sensibility rate of $92 \%$ (when compared with other imagistic studies - CT- MRI which had a sensibility rate of only $60 \%)(10)$

\section{Postoperative outcomes}

In a retrospective study conducted on 54 patients surgically treated between January 1990 and August 2009 for recurrent or persistent gynecological malignancies Benn et all tried to identify the most important prognostic factors associated with a higher rate of survival. At the moment of entering the study $34 \%$ of patients had been submitted to neo-adjuant irradiation, $26 \%$ to neo-adjuvant chemotherapy and secondary to radiotherapy while $12 \%$ of cases benefited from adjuvant oncological treatment.

In $91 \%$ of cases the indication was the persistence of a recurrent tumor. The median disease free survival was 32 months while the most frequent site of recurrence was the vaginal stump. Surgery varied between total pelvic exenteration ( $67 \%$ of cases), anterior exenteration ( $24 \%$ of cases) and posterior exenteration ( $9 \%$ of cases)

The early postoperative complications were seen in $50 \%$ of patients and consisted in myocardial ischemia, pneumonia, deep venous thrombosis while the late postoperative complications were represented by bowel obstruction or pyelonephritis and were seen in up to $61 \%$ of cases.

The mean follow-up time was 12,5 months while the mean overall survival was 29 months. One year overall survival was $64 \%$.

A higher overall survival was seen in younger patients (at the moment of recurrence's diagnosis), with lower postoperative complications and negative margins of resection; they also demonstrated that cases with recurrent cervical cancer had a higher overall survival when compared to those with vulvar or vaginal cancer. (11)

Other studies have shown that the overall survival for patients which are submitted to pelvic exenteration for gynecological tumors is lower when compared to those in whom the primary tumor is a rectosigmoidian one.

A retrospective study conducted on 50 patients surgically treated between 1996 and 2011 revealed higher rates of survival in cases revealed an overall survival of 24 months in cases with locally invasive rectal cancer and only 18 months for those with cervical cancer.(4)

Other primary pelvic tumors which might be amenable to palliative pelvic exenteration are represented by advanced ovarian cancer, bladder or prostate cancer but these are less frequently encountered due to the higher tendency of producing distant metastases associated with a lower rate of local pelvic invasion. (12)

Nowadays the most important sources of early postoperative complications responsive for an increased postoperative morbidity and mortality are related to the apparition of urinary or gastrointestinal fistulas and to the management of pelvic remnant dead space

Table 1 - Main studies regarding the effectiveness of palliative pelvic exenteration

\begin{tabular}{|c|c|c|c|c|c|c|c|}
\hline Clinical study & $\begin{array}{l}\text { No. of } \\
\text { patients }\end{array}$ & $\begin{array}{l}\text { Primary } \\
\text { tumor }\end{array}$ & $\begin{array}{l}\text { Symptoms leading } \\
\text { to palliation }\end{array}$ & $\begin{array}{c}\text { Symptoms } \\
\text { relief }\end{array}$ & $\begin{array}{c}\text { Postoperative } \\
\text { morbdity }\end{array}$ & $\begin{array}{c}\text { Postoperative } \\
\text { mortality }\end{array}$ & $\begin{array}{c}\text { OS } \\
\text { (months) }\end{array}$ \\
\hline Brophy et al (13) & 35 & $\begin{array}{l}21 \text { colorectal } \\
9 \text { urinary } \\
5 \text { gynecologic }\end{array}$ & $\begin{array}{c}\text { Pain (12 cases) } \\
\text { bleeding (11 cases) } \\
\text { fistula ( } 7 \text { cases) } \\
\text { obstruction ( } 6 \text { cases) }\end{array}$ & $88 \%$ & $47 \%$ & $3 \%$ & 20 \\
\hline Woodhouse et al (14) & 14 & & - & $58 \%$ & - & - & 17 \\
\hline Khurt et al (15) & 53 & $\begin{array}{l}36 \text { colorectal } \\
17 \text { gynecologic }\end{array}$ & - & & $\begin{array}{c}86 \% \\
\text { (colorectal group) } \\
76 \% \\
\text { (gynecologic group) }\end{array}$ & 0 & $\begin{array}{c}10,7 \\
\text { (colorectal } \\
\text { group) } \\
7 \\
\text { (gynecologic } \\
\text { group) }\end{array}$ \\
\hline Asish et al (17) & 14 & Prostate cancer & Pain & $79 \%$ & 50 & 0 & 24 \\
\hline Kakuda et al (16) & 5 & Rectal cancer & - & - & - & - & 8 months \\
\hline
\end{tabular}


which constitutes after resection. In cases in which a palliative procedure is performed these risk factors are even more dangerous than in patients submitted to radical surgery. This is related to the fact the patients who undergo palliative surgery usually have a long history of neoplastic disease associated with recurrent cycles of chemo-irradiation, denutrition and anemia.

\section{CONCLUSIONS}

Pelvic exenteration represents a difficult surgical procedure associated with high risks of postoperative complications. Once the surgical technique and postoperative management improved the overall survival increased. However there are still cases diagnosed in an advanced stage of the disease presenting distant metastases or local unresectable invasion associated with debilitating symptoms. In these cases palliative exenteration is needed in order to improve the quality of life.

\section{REFERENCES}

1. Eifel PJ, Berek JS, Thigpen JT. Cancer of the cervix, vagina and vulva. In: DeVita VT, Hellman S, Rosenberg SA, eds. Cancer Principles and Practice of Oncology. 5th ed. Philadelphia, Pa: Lippincott Williams \& Wilkins; 1997:1433-78

2. Brunschwig,A. 1948. Complete excision of pelvic viscera for advanced carcinoma; a one-stage abdominoperineal operation with end colostomy and bilateral ureteral implantation into the colon above the colostomy. Cancer 1:177-183.

3. Goldberg,G.L., Sukumvanich,P., Einstein,M.H., Smith,H.O., Anderson,P.S. and Fields,A.L. 2006. Total pelvic exenteration: the Albert Einstein College of Medicine/Montefiore Medical Center Experience (1987 to 2003). Gynecol. Oncol. 101:261-268.

4. Ramamurthy,R., and Duraipandian,A. 2012. Morbidity and outcome of pelvic exenteration in locally advanced pelvic malignancies. Indian J. Surg. Oncol. 3:231-235

5. Hockel,M. 2003. Laterally extended endopelvic resection. Novel surgical treatment of locally recurrent cervical carcinoma involving the pelvic side wall. Gynecol. Oncol. 91:369-377.

6. Morris,M., Alvarez,R.D., Kinney,W.K., and Wilson,T.O. 1996. Treatment of recurrent adenocarcinoma of the endometrium with pelvic exenteration. Gynecol. Oncol. 60:288-291.

7. Barakat,R.R., Goldman,N.A., Patel,D.A., Venkatraman,E.S., and Curtin,J.P. 1999. Pelvic exenteration for recurrent endometrial cancer. Gynecol. Oncol. 75:99-102.

8. Popovich,M.J., Hricak,H., Sugimura,K., and Stern,J.L. 1993. The role of MR imaging in determining surgical eligibility for pelvic exenteration. AJR Am. J. Roentgenol. 160:525-531.

9. Donati,O.F., Lakhman,Y., Sala,E., Burger,I.A., Vargas,H.A., Goldman,D.A., Andikyan,V., Park,K.J., Chi,D.S., and Hricak,H. 2013. Role of preoperative MR imaging in the evaluation of patients with persistent or recurrent gynaecological malignancies before pelvic exenteration. Eur. Radiol. 23: 2906-2915.

10. Lai,C.H., Huang,K.G., See,L.C., Yen,T.C., Tsai,C.S., Chang,T.C., Chou,H.H., Ng,K.K., Hsueh,S., and Hong,J.H. 2004. Restaging of recurrent cervical carcinoma with dual-phase [18F]fluoro-2-deoxy-D-glucose positron emission tomography. Cancer 100:544-552.

11. Benn,T., Brooks,R.A., Zhang,Q., Powell,M.A., Thaker,P.H., Mutch,D.G., and Zighelboim,l. 2011. Pelvic exenteration in gynecologic oncology: a single institution study over 20 years. Gynecol. Oncol. 122:14-18.

12. Chen,H.S., and Sheen-Chen,S.M. 2001. Total pelvic exenteration for primary local advanced colorectal cancer. World J. Surg. 25:1546-1549.

13. Brophy,P.F., Hoffman,J.P., and Eisenberg,B.L. 1994. The role of palliative pelvic exenteration. Am. J. Surg. 167:386-390.

14. Woodhouse,C.R., Plail,R.O., Schlesinger,P.E., Shepherd,J.E., Hendry,W.F., and Breach,N.M. 1995. Exenteration as palliation for patients with advanced pelvic malignancy. Br. J. Urol. 76:315-320.

15. Kuhrt,M.P., Chokshi,R.J., Arrese,D., and Martin,E.W., Jr. 2012. Retrospective review of pelvic malignancies undergoing total pelvic exenteration. World J. Surg. Oncol. 10:110.

16. Kakuda,J.T., Lamont,J.P., Chu,D.Z., and Paz,I.B. 2003. The role of pelvic exenteration in the management of recurrent rectal cancer. Am. J. Surg. 186:660-664. 